\title{
Specific Heat Study of Superconductivity in $\mathrm{Cu}_{0.061} \mathrm{TiSe}_{2}$
}

\author{
J. KaČmarČík ${ }^{a, *}$, Z. Pribulová ${ }^{a}$, V. Pal'uchová ${ }^{a}$, P. Husaníková ${ }^{b}$, G. Karapetrov $^{c}$, \\ V. KOMANICKÝ ${ }^{a}$, P. SAMUELY ${ }^{a}$ \\ ${ }^{a}$ Centre of Low Temperature Physics @ Institute of Experimental Physics, Slovak Academy of Sciences \& P.J. \\ Šafárik University, Park Angelinum 9, 04001 Košice, Slovakia \\ ${ }^{b}$ Institute of Electrical Engineering, Slovak Academy of Sciences, Dúbravská cesta 9, 84104 Bratislava, Slovakia \\ ${ }^{c}$ Department of Physics, Drexel University, 3141 Chestnut St., Philadelphia, PA 19104, USA
}

In $\mathrm{TiSe}_{2}$ a charge-density-waves order is continuously suppressed by copper doping and superconductivity appears. We report a calorimetric study performed on the superconducting single crystal of $\mathrm{Cu}_{0.061} \mathrm{TiSe}_{2}$ with $T_{c}=2.8 \mathrm{~K}$, measured by the ac technique at low temperatures down to $0.6 \mathrm{~K}$ and magnetic fields up to $2 \mathrm{~T}$. Sharp specific heat anomaly at the superconducting transition is found. The temperature dependence of the electronic specific heat and the angular dependence of the critical temperature, measured at a fixed magnetic field, consistently indicate on the existence of a single s-wave superconducting gap with a coupling strength of $2 \Delta / k_{B} T_{c}=3.6$, close to BCS limit.

DOI: $10.12693 /$ APhysPolA.126.322

PACS: 72.80.Ga, 74.25.Bt, 71.45.Lr

\section{Introduction}

$1 T$ - $\mathrm{TiSe}_{2}$ is a layered compound with trigonal symmetry [1]. In $\mathrm{TiSe}_{2}$ layers the Ti atoms are in octahedral coordination with Se. The layers are bonded to each other by van der Waals forces. Undoped $\mathrm{TiSe}_{2}$ is the oldest known system with charge density waves (CDW), a quite frequent effect in transition metal dichalcogenides. Electron and X-ray diffraction studies show the presence of reflections corresponding to the basic trigonal structure and also superstructure reflections associated with the CDW state with doubled lattice constants [2]. As $\mathrm{Cu}$ atoms are added, they occupy positions between the $\mathrm{TiSe}_{2}$ layers and expand the $c$-lattice parameter. In the resulting $\mathrm{Cu}_{x} \mathrm{TiSe}_{2}$ a tunable transition from CDW to superconductivity has been discovered [3]. Superconductivity starts at about $x=0.04$, while the transition temperature to CDW state is decreased from 200 to $0 \mathrm{~K}$ at about $x=0.06$ (quantum critical point). The superconducting $T_{c}$ has a maximum of $4.15 \mathrm{~K}$ at about $x=0.08$ and then slightly decreases up to solubility limit at $x=0.11$. The interplay between the two competing orders is of high interest. One of the issues is a possible unconventional superconductivity in such systems. Hillier et al. [4] suggested from their muon spectroscopy measurements that a polycrystalline $\mathrm{Cu}_{0.06} \mathrm{TiSe}_{2}$ sample is characterized by a single $s$-wave superconducting gap $\Delta$ with a reduced value $2 \Delta / k_{B} T_{c}=2.5$, much smaller than the BCS canonical value of 3.52 , possibly indicating a multigap superconductivity. We addressed the characteristics of the superconducting gap by the specific heat measurements on a single crystal with a similar copper

*corresponding author; e-mail: kacmarci@saske.sk doping. We find that the temperature dependence of the electronic specific heat can be well described within the BCS framework with a single superconducting gap of medium strength $2 \Delta / k_{B} T_{c}=3.6$. The angular dependence of the critical temperature in a magnetic field is consistent with this conclusion.

\section{Measurements}

The specific heat measurements were performed using an ac technique [5] at temperatures down to $0.6 \mathrm{~K}$ and in magnetic fields up to $2 \mathrm{~T}$. Ac calorimetry is based on applying periodically modulated power and measuring resulting temperature oscillations of the sample in a proper frequency range. It is very sensitive to relative changes on minute samples and enables continuous temperature and field measurements. The crystal was prepared via the iodine vapor transport method [6]. The energy-dispersive spectroscopy analysis yields a copper content of 1.98 at.\%, which corresponds to $x=0.0605$ in the $\mathrm{Cu}_{x} \mathrm{TiSe}_{2}$ formula. The superconducting critical temperature of the sample is $T_{c}=2.8 \mathrm{~K}$.

\section{Results and discussion}

Figure 1 presents the superconducting electronic specific heat divided by temperature which was obtained from the zero-field measurement of $C(T)$ by subtracting the normal state specific heat $C_{n}(T)$ taken at $2 \mathrm{~T}$, i.e. $\Delta C(T) / T=C(T) / T-C_{n}(T) / T$. The thermodynamic transition temperature $T_{c}$ at zero field was determined from the local entropy balance around the phase transition, giving $T_{c}=2.8 \mathrm{~K}$. It is known that the parameter $\Delta C\left(T_{c}\right) / \gamma T_{c}$ ( $\gamma$ is the Sommerfeld coefficient) can be used to measure the strength of the electron coupling.

Taking the height of the jump at anomaly as 1.6 and $\gamma=-1$, both in arbitrary units, we obtain the ratio of 
$\Delta C\left(T_{c}\right) / \gamma T_{c}$ of approximately 1.6, which is slightly larger than that for the BCS weak coupling limit (1.43).

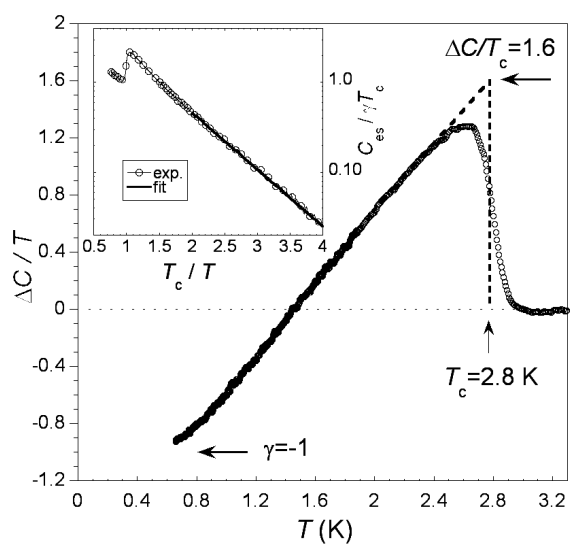

Fig. 1. Electronic specific heat in zero magnetic field. Dashed line is entropy conservation construction around critical temperature. Inset: exponential dependence of the specific heat, full line represents the best fit.

At very low temperatures the electronic specific heat of a single gap superconductor shows an activation behaviour over the superconducting energy gap $C(T) \sim$ $\exp \left(-\Delta / k_{B} T\right)$. For the weak coupling BCS limit with $2 \Delta / k_{B} T_{c}=3.52, C(T) / \gamma T_{c} \sim \exp \left(-1.76 T_{c} / T\right)$. In the temperature range $2.5<T_{c} / T<4.5$ the exact formula reads as $C(T) / \gamma T_{c}=8.5 \exp \left(-1.44 T_{c} / T\right)$ [7]. The inset of Fig. 1 displays the logarithm of the electronic specific heat versus $T_{c} / T$ showing clear exponential behaviour. The best fit yields a slope of $-1.46 T_{c} / T$, indicating slightly enhanced coupling strength $2 \Delta / k_{B} T_{c}=3.6$. This is a direct proof that $\mathrm{Cu}_{x} \mathrm{TiSe}_{2}$ with $x=0.0605$ is a single gap superconductor close to BCS limit.

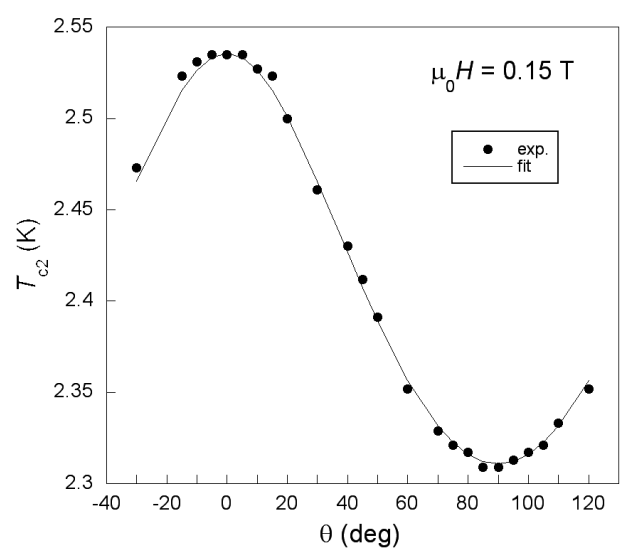

Fig. 2. Angular dependence of transition temperature $T_{c 2}$ in magnetic field of $0.15 \mathrm{~T}$.

Figure 2 presents the results of the transition temperatures $T_{c 2}$ measured at a fixed magnetic field of $\mu_{0} H=$ $0.15 \mathrm{~T}$ oriented at different angles $\theta$ with respect to the $a b$ basal planes of the sample. It has been extracted from the temperature sweeps of the specific heat measurements in the same way as in Fig. 1. The anisotropy of a single gap BCS superconductor is described by the effective mass model within the Ginzburg-Landau theory with $T_{c 2}(\theta)=$ $T_{c 0}+H\left(\cos ^{2}(\theta)+\Gamma^{2} \sin ^{2}(\theta)\right)^{0.5} /\left(d H_{c 2}^{a b} / d T\right)$, where $T_{c 0}$ is the zero-field transition temperature, $\Gamma=H_{c 2}^{a b} / H_{c 2}^{c}$ is the superconducting anisotropy parameter, equal to the ratio of the upper critical fields for the field parallel and perpendicular to the $a b$ planes respectively, and $d H_{c 2}^{a b} / d T$ is the slope of upper critical field near $T_{c 0}$. As can be seen this formula describes our data well. Such behaviour is in strong contrast with another transition metal dichalcogenide, namely $\mathrm{NbS}_{2}$ which is a two gap system with one gap much smaller than BCS value [8]. For $\mathrm{NbS}_{2}$ a significant deviation from the effective mass model is found [9], similarly like in the case of other multigap superconductors: $\mathrm{MgB}_{2}[10]$ and iron pnictides [11].

\section{Conclusions}

The specific heat measurements on the singlecrystaline $\mathrm{Cu}_{0.061} \mathrm{TiSe}_{2}$ with $T_{c}=2.8 \mathrm{~K}$ provide a strong evidence that despite proximity to the charge-densitywave order the system is a conventional single $s$-wave superconductor close to BCS limit.

\section{Acknowledgments}

This work was supported by ERDF EU under the contract No. ITMS26220120005, VEGA No. 2-0135-13, and MP1201 COST Action. Liquid nitrogen has been sponsored by the US Steel Košice.

\section{References}

[1] I. Oftedal, Z. Phys. Chem. 134, 301 (1928).

[2] K.C. Woo, F.C. Brown, W.L. McMillan, R.J. Miller, M.J. Schaffman, M.P. Sears, Phys. Rev. B 14, 3242 (1976).

[3] E. Morosan, H.W. Zandbergen, B.S. Dennis, J.W.G. Bos, Y. Onose, T. Klimczuk, A.P. Ramirez, N.P. Ong, R.J. Cava, Nat. Phys. 2, 544 (2006).

[4] A.D. Hillier, P. Manuel, D.T. Adroja, J.W. Taylor, A.K. Azad, J.S. Irvine, Phys. Rev. B 81, 092507 (2010).

[5] P.F. Sullivan, G. Seidel, Phys. Rev. 173, 679 (1968).

[6] C.S. Oglesby, E. Bucher, C. Kloc, H. Hohl, J. Cryst. Growth 137, 289 (1994).

[7] R. D. Parks, Superconductivity, Marcel Dekker, New York, 1969.

[8] J. Kačmarčík, Z. Pribulová, C. Marcenat, T. Klein, P. Rodière, L. Cario, P. Samuely, Phys. Rev. B 82, 014518 (2010).

[9] Z. Pribulová, M. Leroux, J. Kačmarčík, C. Marcenat, T. Klein, P. Rodière, L. Cario, P. Samuely, Acta Phys. Pol. 118, 1024 (2010).

[10] P. Szabó, P. Samuely, J. Kačmarčík, L. Lyard, T. Klein, J. Marcus, C. Marcenat, Czechoslovak J. Phys. 54, 449 (2004).

[11] U. Welp, R. Xie, A.E. Koshelev, W.K. Kwok, H.Q. Luo, Z.S. Wang, G. Mu, H.H. Wen, Phys. Rev. B 79, 094505 (2009). 